\title{
DOENÇAS FOLIARES, CANCRO E NÚMERO DE FRUTOS RELACIONADOS COM A ADUBAÇÃO NITROGENADA EM PESSEGUEIRO ${ }^{1}$
}

\author{
SILVANA REGINA DE SOUZA², LOUISE LARISSA MAY DE MIO ${ }^{3}$, BEATRIZ MONTE SERRAT ${ }^{4}$, \\ MARCIO ALBERTO CHALLIOL ${ }^{5}$
}

RESUMO - Para a implementação da Produção Integrada (PI) de Pêssegos no Paraná foram necessárias algumas adaptações regionais no manejo da cultura. A recomendação de adubação nitrogenada para o Rio Grande do Sul determinava, em 2003, um máximo anual de $80 \mathrm{~kg} / \mathrm{ha}$ de nitrogênio $(\mathrm{N})$, o que foi considerado um fator limitante para as altas produtividades obtidas por alguns produtores no Estado do Paraná. Por outro lado, o excesso de nitrogênio pode elevar a incidência de doenças de folhas e de ramos, prejudicando o desenvolvimento das plantas. $O$ presente trabalho objetivou avaliar o efeito da adubação nitrogenada na produção e em três doenças de pessegueiro cultivado em sistema de PI. O experimento foi realizado no município da Lapa, com a cultivar Chimarrita, com três tratamentos $(40,80$ e $160 \mathrm{~kg} / \mathrm{ha} \mathrm{de} \mathrm{N})$ e seis repetições. A produção foi avaliada contando-se o número de frutos totais por planta útil na colheita. Para avaliação das doenças, foi determinado o número de lesões (cancros) de Botryospheria dothidea em uma planta marcada por parcela em dois anos consecutivos (2003 e 2004), no período pós-floração. Para furo de bala (Wilsonomyces carpophilus) determinouse a incidência da doença no ano de 2002 e para ferrugem (Tranzschelia discolor) avaliou-se incidência e severidade em 2003 e 2004. Com os dados de severidade da ferrugem no tempo foi obtida a curva de progresso da doença e o índice da desfolha no período. No terceiro ano observou-se que na dose de $160 \mathrm{~kg} / \mathrm{ha}$ de $\mathrm{N}$ o número de frutos foi estatisticamente significativo e maior que nos demais tratamentos. A incidência de cancros aumentou entre os anos, porém não houve diferenças significativas entre as dosagens de nitrogênio, o furo de bala também não foi influenciado pelos tratamentos. Aárea abaixo da curva de progresso da doença (AACPD) para severidade de ferrugem foi 20,7 \% superior na menor dose em relação a maior dose de nitrogênio, entretanto, sem reflexos sobre a desfolha.

Termos para indexação: Pêssego, nitrogênio, furo de bala, ferrugem e cancro.

\section{LEAVE DISEASES, CANKER AND NUMBER OF FRUITS RELATED WITH THE NITROGEN FERTILIZER IN PEACH TREES}

\begin{abstract}
With the introduction of an integrated production of peaches (PI) in Paraná, some modifications during the handling of this culture were necessary, in order to adapt it to the regional conditions. The Nitrogen fertilizer was recommended to PI from Rio Grande do Sul and it stated a maximum of $80 \mathrm{~kg} /$ ha of nitrogen $(\mathrm{N})$ annually, becoming a limiting factor to the high productivity reached by some producers in the State of Parana. On the other hand, the increasing of the nitrogen dosage may favor a major incidence of leave and branch diseases, damaging the development of the plants. The present work aimed to evaluate the effect of nitrogen fertilizer in the production and in three diseases, cultivated in the PI system. An experiment in Lapa was carried through with the Chimarrita Cultivar, with three treatments $(40,80$ and $160 \mathrm{~kg} / \mathrm{ha}$ of $\mathrm{N})$ and six repetitions. The production was evaluated counting the number of total fruits for useful plant in the harvest. For evaluation of the diseases the number was determined by the number of lesions (cankers) of Botryosphaeria dothidea in a marked plant of each fragment, two years in a row (2003 and 2004), in the period after flowering, shot hole (Wilsonomyces carpophilus) was determined by the incidence of the disease in the year of 2002 and to rust (Tranzschelia discolor) it was evaluated the incidence and severity in 2003 and 2004. With the data of the rust severity the progress curve of the disease and the fall of the leaves index in the period were obtained. In the third year it was observed that in the dose of $160 \mathrm{~kg} / \mathrm{ha}$ of N the number of fruits was significant and bigger than in the other treatments. It was observed the growth of the incidence of cankers among the years, however without significant result for the different dosages of nitrogen. The shot hole also was not influenced by the treatments. The area below the progress curve of the diseases (AACPD) for rust severity was $20,7 \%$ superior to the minor dose in relation to the major dose of nitrogen, however without any reflexes over the fall of the leaves index.
\end{abstract}

Index Terms: Peach, nitrogen, shot role, rust e canker.

\footnotetext{
${ }^{1}$ Trabalho (111-06) . Recebido em 03-08-2006. Aceito para publicação em: 14-06-2007.

${ }^{2}$ Mestra formada pelo curso de Pós graduação do departamento de Solos e Engenharia Agrícola da Universidade Federal do Paraná, R. da Bandeira, 415, apto 31 C. Cep: 80035-050. Tel.: (41)3252-6965. E-mail: souuza@pop.com.br.

${ }^{32}$ Professora Dra do Departamento de Fitotecnia e Fitossanitarismo, Setor de Ciências Agrárias da Universidade Federal do Paraná, R. dos Funcionários 1540, 80035-050. Tel.: (41) 3350-5736. E-mail: maydemio@ufpr.br

${ }^{4}$ Professora Dra do Departamento de Solos e Engenharia Agrícola, Setor de Ciências Agrárias da Universidade Federal do Paraná, R. dos Funcionários 1540, 80035-050. Tel.: (41) 3350-5609. E-mail: bmserrat@ufpr.br

${ }^{5}$ Eng $^{\text {o }}$. Agrônomo formado pela Universidade Federal do Paraná, Setor de Ciências Agrárias. E-mail: marciochalliol@yahoo.com.br
} 


\section{INTRODUÇÃO}

Com a introdução da Produção Integrada (PI) de pêssegos no Paraná, algumas modificações no manejo da cultura foram necessárias, exigindo estudos para as recomendações nas condições do Estado. Uma das proposições está relacionada à adubação nitrogenada, para a qual as Normas de Produção Integrada de Pêssego (2001) determinavam um máximo anual de $80 \mathrm{~kg} / \mathrm{ha}$ de N. No entanto, segundo alguns produtores, a referida recomendação tornou-se fator limitante para a manutenção dos valores de produtividade no Estado do Paraná. No município da Lapa, por exemplo, a produtividade tem alcançado $20 \mathrm{t} / \mathrm{ha}$ e, em Araucária 35 t/ha (Fachinello et al., 2004).

Para o Paraná, são escassas as informações sobre a relação entre doses de adubo nitrogenado e ocorrência de doenças em pessegueiro. O manejo do nitrogênio é, muitas vezes, limitante e, de acordo com Van Raij (1991), enquanto adubações elevadas podem proporcionar aumento na suscetibilidade das plantas a doenças, a deficiência na quantidade desse elemento pode ocasionar a redução na produção de frutos, de acordo com a afirmação de Kishino et al. (1978) e Yamada (2004). Todavia, Marschner (1995) comenta que todos os fatores que favorecem as atividades metabólicas e de síntese de células das hospedeiras (como a adubação nitrogenada), também corroboram a resistência das plantas a algumas doenças. Assim, cada região terá um ponto de equilíbrio entre a adubação nitrogenada e a qualidade fitossanitária dos pomares.

Vários autores relacionam o incremento de nitrogênio à maior incidência de doenças (Soave et al., 1977; Tanaka et al., 2000; Rodrigues et al., 2002; Taylor \& Van Raij, 2003) para diferentes culturas. Para rosáceas de caroço, os trabalhos são ainda incipientes. Dentro do conceito de PI, é fundamental o desenvolvimento de tecnologias que consideram as relações nutricionais com a produtividade e também com fatores fitossanitarios, tais como a ocorrência das diferentes doenças ao longo do ciclo da cultura (Fachinello et al., 2004). Diferentes doses de nitrogênio podem interferir tanto na severidade quanto na incidência de doenças, com reflexos sobre a desfolha da planta e, conseqüentemente, sobre a produtividade (Tutida, 2006 e Pereira et al. 1996).

Para o pessegueiro, a principal doença é a podridão-parda, entretanto doenças foliares, como furo-de-bala (Wilsonomyces carpophilus) e ferrugem (Tranzschelia discolor) e também cancro (Botryosphaeria dothidea), têm aumentado nos últimos ciclos nas condições de produção do Estado do Paraná (May De Mio et al., 2004).

O objetivo do trabalho foi avaliar o efeito de doses de nitrogênio sobre a produção, sobre doenças foliares (furo-debala e ferrugem) e sobre cancro em pessegueiros cultivados sob sistema de PI, durante três ciclos, na região da Lapa-PR.

\section{MATERIAL E MÉTODOS}

O experimento foi realizado no município da Lapa-PR, sob clima $\mathrm{Cfb}$ (subtropical úmido) e temperatura média de $20,4^{\circ} \mathrm{C}$ no verão e $12,7^{\circ} \mathrm{C}$ no inverno. Foi instalado em 2002 , em pomar da cultivar "chimarrita" com três anos de idade, e foi conduzido durante três safras, 2002-2003, 20032004 e 20042005. O manejo fitossanitário foi efetuado conforme as Normas da Produção Integrada de Pêssego (2003), sendo que foram feitas pulverizações de captana, mancozebe e captana + fosfito de $\mathrm{K}$, no período de julho a outubro para furo-de-bala e, após a colheita, mancozebe e tebuconazole no período de dezembro a março para ferrugem, excluindo-se os tratamentos de inverno (realizados com calda bordaleza).

Os tratamentos foram representados por doses de adubo nitrogenado na forma de uréia: 40; 80 e 160 kg/ha de nitrogênio, com seis repetições. Cada parcela experimental foi composta por seis plantas, considerando-se a planta central como parcela útil. A adubação nitrogenada foi realizada em três etapas: no início da brotação, após o raleio e após a colheita. As adubações fosfatada e potássica foram realizadas nas doses e épocas conforme Dolinski et al. (2005).

A produção foi avaliada contando-se o número de frutos totais por planta útil, na colheita.

\section{Avaliação das doenças}

Para a avaliação do cancro, foi determinado o número de lesões na parcela útil, sendo realizada em uma única data, nos anos de 2003 e 2004, no período pós-floração, seguindo metodologia descrita em Monteiro et al. (2004).

O furo-de-bala foi avaliado por incidência nas folhas, em dois ramos do ano, marcados/planta, em novembro de 2002.

A avaliação da ferrugem foi realizada em duas safras, na primeira, em 2003, foi realizada avaliação da severidade da doença no final da epidemia, utilizando-se da escala diagramática de notas desenvolvida por Martins (1994), considerando-se dois ramos marcados/planta, com 10 níveis de severidade, de 1 a 10 , correspondendo a 0,$06 ; 0,09 ; 0,19 ; 0,28 ; 0,50 ; 0,85 ; 1,48 ; 2,52 ; 4,22$ e $6,97 \%$ da área foliar afetada pela doença. Em 2004, avaliaram-se a incidência e a severidade da ferrugem, em quatro ramos do ano/ planta, distribuídos em quatro quadrantes da planta, de janeiro a abril. Foram realizadas três avaliações: uma no início da epidemia (10 de fevereiro) e outras duas aos 28 e 42 dias após a primeira. Com os dados do progresso da doença no campo, foi calculada a área abaixo da curva de progresso da doença (AACPD) para incidência e severidade, de acordo com metodologia descrita por Campbell \& Madden (1990).

Os resultados foram submetidos à análise de variância, e as médias, comparadas pelo teste de tukey a 5\% de significância. Foram também realizadas correlações ente os tratamentos e as variáveis, utilizando-se do programa Statistica $6.0^{\circledR}$.

\section{RESULTADOS E DISCUSSÃO}

Durante todas as avaliações e coletas realizadas, não foram detectados sintomas visuais de deficiência nutricional em nenhuma das plantas do experimento.

Para produção, nos dois primeiros anos avaliados, não houve diferença significativa entre os tratamentos, mas no terceiro ano, na dose de $160 \mathrm{~kg} /$ ha de $\mathrm{N}$, as plantas produziram em média 
260 frutos a mais que nos demais tratamentos (Tabela 1). Na mesma área, Dolinski et al. (2005), avaliando produtividade da parcela total, concluíram que a redução na adubação nitrogenada afetou negativamente o número de frutos.

Com relação ao efeito da adubação nitrogenada sobre o cancro, verificou-se que não ocorreram diferenças significativas entre os tratamentos, entretanto observou-se um incremento de $40 \%$ de um ano para o outro. A incidência variou em média de 0,3 a 6,5 no primeiro ano e de 40 a 47 cancros/planta no segundo (Tabela 2 ). O incremento da doença no ano de 2004, pode ter sido causado pelo clima atípico ocorrido durante o experimento, como, por exemplo, a chuva de granizo do final da safra de 2003. Os ferimentos provocados na planta podem ter facilitado a entrada do patógeno; além disso, embora tenham sido eliminados os ramos infectados durante o inverno, o processo de poda e desinfestação de ferramentas não foram suficientes para conter o avanço da doença. Os procedimentos de desinfestação são recomendados para prevenir a entrada do fungo pelos ferimentos, evitando que o fungo sobreviva nos tecido durante o inverno (Jenkins \& Reinganum, 1965; Carvalho, 1980; Ogawa et al., 1995; Bleicher, 1997).

Para furo-de-bala, a incidência foi de 50 a $52 \%$, não apresentando correlação com as dosagens de nitrogênio (Tabelas 2 e 4 ). A severidade estava baixa e não foi considerada, assim, a doença não foi mais avaliada nos anos seguintes, pois, não tem tido relação com a produção nas condições do Paraná, segundo observações de May De Mio et al. (2004). Na literatura internacional, os trabalhos com esta doença são escassos. No Brasil, pouco tem sido publicado sobre a doença em rosáseas de caroço. Challiol et al. (2006), monitorando doenças em experimento de PI, citam que o furo-de-bala, em plantas de pessegueiro fertilizadas com $80 \mathrm{~kg} /$ ha de $\mathrm{N}$ na Lapa-PR, não causou problema, mantendo-se com baixa severidade. Em contrapartida, Tutida (2006), trabalhando com ameixeira na região de Araucária-PR, encontrou relação entre a doença furo-de-bala e nitrogênio, em condições de alta incidência (93\%) e severidade, sendo a doença mais severa a partir de adubações superiores a $160 \mathrm{~kg} / \mathrm{ha}$ de $\mathrm{N}$.

Para ferrugem, em 2003, a epidemia foi muito severa e, em fevereiro, atingia notas que variavam de 8,8 a 9,2 (dados não apresentados), não diferindo entre os tratamentos. Em 2004, a incidência não diferiu entre os tratamentos, e a severidade da doença apresentou redução com o aumento da dose de nitrogênio, cujos valores da área abaixo da curva de progresso da doença foram de 124,6, 108,4 e 98,8, respectivamente, para as doses de 40; 80 e $160 \mathrm{~kg} / \mathrm{ha}$ de $\mathrm{N}$, e isso pode ser indício de desequilíbrio nutricional das plantas na menor dose de nitrogênio. Essa diferença entre os tratamentos foi evidenciada principalmente nas avaliações realizadas de 09 a 23-03 (Tabela 3). Pereira et al. (1996) obtiveram resultados semelhantes para a cultura do café, onde a adubação nitrogenada, em geral, reduziu significativamente a área foliar lesionada por ferrugem em relação à testemunha.

Nos pomares do Estado do Paraná, o limite superior da dose de nitrogênio, segundo a recomendação das Normas de Produção Integrada de Pêssego (2001), poderá ser ampliado, sendo que houve diferença na produção, em número de frutos, onde, no último ano do experimento, ocorreu maior produção na dose de $160 \mathrm{~kg} /$ ha de $\mathrm{N}$ e também em relação à ferrugem, onde a menor dose do fertilizante, $40 \mathrm{~kg} / \mathrm{ha}$, refletiu em maior severidade da doença. No entanto, há necessidade de experimentos com maiores dosagens para a verificação do limite entre deficiência e excesso de nitrogênio para as plantas, em função de doenças.

A desfolha das plantas não diferiu entre os tratamentos. (dados não apresentados). Esse fato é positivo, pois a ferrugem acarreta queda precoce das folhas a partir de fevereiro, que estimula a planta a emitir uma nova brotação, a qual é perdida na época de desfolha natural (maio). Esse gasto desnecessário de energia, por parte da planta, resultaria na diminuição da produção na safra seguinte (Margarido, 1988). Challiol et al. (2006) observaram que o aumento da severidade da ferrugem foi acompanhado da desfolha, entretanto, no experimento em questão, não foi detectada relação entre a severidade da doença e a desfolha das plantas nos tratamentos.

Para uma investigação mais aprofundada sobre a interferência do nitrogênio na desfolha, recomenda-se que o estudo seja realizado em condições naturais da epidemia, sem controle com fungicidas.

Nas análises de correlação realizadas entre as variáveis: dosagens de N x produção (2002, 2003 e 2004), AACPD para severidade e incidência em ferrugem (2004), incidência de furode-bala (2002) e incidência de cancro (2002 e 2003), encontrou-se correlação somente na variável N x produção de 2004 (Tabela 4).

TABELA 1 - Influência de dosagens de nitrogênio no número de frutos colhidos por planta de pessegueiro, em três safras. Lapa-PR.

\begin{tabular}{cccc}
\hline Tratamento kg/ha & \multicolumn{3}{c}{ Produção (número de frutos/planta) } \\
\cline { 2 - 4 } de nitrogênio & $\mathbf{2 0 0 2}$ & $\mathbf{2 0 0 3}$ & $\mathbf{2 0 0 4}$ \\
\hline $\mathbf{4 0}$ & $231,4^{\mathrm{ns}}$ & $379,2^{\mathrm{ns}}$ & $313,6 \mathrm{~b}$ \\
$\mathbf{8 0}$ & 214,8 & 339,2 & $312,8 \mathrm{~b}$ \\
$\mathbf{1 6 0}$ & 213,4 & 469,2 & $572,4 \mathrm{a}$ \\
\hline C.V.\% & 29,1 & 25,5 & 37,7 \\
\hline
\end{tabular}

ns - não significativo. Médias seguidas por letras distintas, dentro da mesma coluna, diferem entre si, pelo teste de tukey, ao nível de $5 \%$ de probabilidade.

TABELA 2 - Incidência em porcentagem de folhas com furo de bala ( $W$. carpophilus) em duas datas de avaliação em 2002 e incidência em número de cancros $(B$. dothidea) em dois anos (2003 e 2004) nas plantas de pessegueiro, no município da Lapa - PR.

\begin{tabular}{ccccc}
\hline & \multicolumn{4}{c}{ Incidência (\%) } \\
\cline { 2 - 5 } Tratamento & \multicolumn{2}{c}{ Furo de bala } & \multicolumn{2}{c}{ Cancro } \\
\cline { 2 - 5 } Kg/ha de N & 13.11 .02 & 27.11 .02 & 2003 & 2004 \\
\hline $\mathbf{4 0}$ & $44.4^{\mathrm{ns}}$ & $52.0^{\mathrm{ns}}$ & $6,5^{\mathrm{ns}}$ & $44,7^{\mathrm{ns}}$ \\
$\mathbf{8 0}$ & 44.5 & 50.6 & 0,3 & 40,7 \\
$\mathbf{1 6 0}$ & 43.4 & 50.3 & 3,2 & 47,5 \\
\hline
\end{tabular}

${ }^{1}$ Teste t a $5 \%$ de significância, entre os tratamentos, ${ }^{\text {ns }}$ - não significativo. 
TABELA 3 - Influência de dosagens de nitrogênio na severidade de ferrugem (Tranzschelia discolor) e na área abaixo da curva de progresso da doença (AACPD) em pessegueiro na safra de 2004, Lapa-PR.

\begin{tabular}{ccccc}
\hline Tratamento & \multicolumn{4}{c}{ Severidade $^{(1)}$} \\
\cline { 2 - 5 } kg/ha de N & $\mathbf{0 9 / 0 3 / 0 4}$ & $\mathbf{2 3 / 0 3 / 0 4}$ & $\mathbf{0 6} / \mathbf{0 4} / \mathbf{0 4}$ & $\mathbf{A A C P D}$ \\
\hline $\mathbf{4 0}$ & $2,2 \mathrm{a}$ & $2,7 \mathrm{a}$ & $5,7^{\mathrm{ns}}$ & $124,5 \mathrm{a}$ \\
$\mathbf{8 0}$ & $1,7 \mathrm{ab}$ & $2,3 \mathrm{ab}$ & 5,6 & $108,4 \mathrm{ab}$ \\
$\mathbf{1 6 0}$ & $1,5 \mathrm{~b}$ & $2,1 \mathrm{~b}$ & 5,1 & $98,8 \mathrm{~b}$ \\
\hline C.V. $\%$ & 26,4 & 16,3 & 12,4 & 16,7 \\
\hline
\end{tabular}

(1) - Severidade avaliada utilizando escala de notas de 0 a 10 . As notas representam as seguintes porcentagens: $1-0,06 ; 2-0,09 ; 3-0,19 ; 4-$ 0,$28 ; 5-0,50 ; 6-0,85 ; 7-1,48 ; 8-2,52 ; 9-4,22 ; 10-6,97$. ns - não significativo. Médias seguidas por letras distintas, dentro da mesma coluna, diferem entre si, pelo teste de Duncan, ao nível de $5 \%$ de probabilidade

TABELA 4 - Parâmetros de correlação entre as dosagens de nitrogênio e variáveis de: produção $(2002,2003$ e 2004), AACPD para severidade e incidência em ferrugem (2004), incidência de furo de bala (2002)e incidência de cancro (2002 e 2003), Lapa - PR.

\begin{tabular}{lcc}
\hline Dosagens de N em relação à: & \multicolumn{2}{c}{ Parâmetros de correlação } \\
\cline { 2 - 3 } & $\mathbf{R}$ & $\mathbf{p}$ \\
\hline Produção 2002 & 0,0045 & 0,9856 \\
Produção 2003 & 0,3039 & 0,2200 \\
Produção 2004 & $0,5167 *$ & 0,0281 \\
Severidade ferrugem 2004 & 0,3226 & 0,1915 \\
Incidência ferrugem 2004 $^{\mathbf{1}}$ & 0,0469 & 0,8531 \\
Incidência furo de bala 2002 $^{*}$ & 0,0723 & 0,7754 \\
Incidência cancro 2002 & 0,2713 & 0,2760 \\
Incidência cancro 2003 $^{2}$ & 0,0652 & 0,7969 \\
\hline
\end{tabular}

* significativo a 5\% de probabilidade pelo teste de Tukey. 1 - Área Abaixo da Curva de Progresso da Doença (AACPD).

\section{CONCLUSÕES}

1-Não houve diferença significativa entre os tratamentos para o número de frutos produzidos nos dois primeiros anos e no terceiro houve maior produção de frutos na dose de $160 \mathrm{~kg} / \mathrm{ha}$ de $\mathrm{N}$.

2-A adubação nitrogenada não interferiu na incidência das doenças cancro e furo-de- bala.

3-No terceiro ano de adubação nitrogenada a dose de 160 $\mathrm{kg} / \mathrm{ha}$ de $\mathrm{N}$ proporcionou a redução da severidade da ferrugem em 20,7 \% em relação ao tratamento onde se utilizou a menor dose de adubo (40 kg/ha de $\mathrm{N})$.

\section{REFERÊNCIAS}

BLEICHER, J. Doenças de rosáceas de caroço. In: KIMATI, H.; AMORIM, L.; BERGAMIM, F.; CAMARGO, L.E.A.; REZENDE, J.A.M. Ed. Manual de fitopatologia: doenças de plantas cultivadas. 3.ed. São Paulo: Ceres, 1997. p. 621-627.

CAMPBELL, C.L.; MADDEN, L.V. Introduction to plant disease epidemiology. New York: John Wiley, 1990.532 p.
CARVALHO, P.C.T. Doenças de rosáceas. In: GALLI, F. Ed. Manual de fitopatologia: doenças de plantas cultivadas. 2. ed. São Paulo: Ceres, 1980. p. 443-458.

CHALLIOL, M.A.; MAY-DE MIO, L.L.; CUQUEL, F.L.; MONTEIRO, L.B.; SERRAT, B.M.; MOTTA,A.C.V.; RIBEIRO JÚNIOR, P.J. Elaboração de escala diagramática para furo de bala e avaliação de doenças foliares em dois sistemas de produção em pessegueiro. Revista Brasileira de Fruticultura, Jaboticabal, v.28, p. 391-396, 2006.

DOLINSKI, M.A.; SERRAT, B.M.; MOTTA, A.C.V.; CUQUEL, F.L.; SOUZA, S.R.; MAY DE MIO, L.L.; MONTEIRO, L.B. Produção, teor foliar e qualidade de frutos de pessegueiro 'chimarrita' em função da adubação nitrogenada, na região da Lapa-PR. Revista Brasileira de Fruticultura, Jaboticabal, v.27, p.295-299, 2005.

FACHINELLO, J.C.; TIBOLA, C.S.; MAY-DE MIO, L.L.; MONTEIRO, L.B. Nutrição mineral de fruteira de caroço. In: MONTEIRO, L.B.; MAY-DE MIO, L.L.; SERRAT, B.M.; MOTTAA.C.; CUQUEL F.L. Fruteiras de caroço: uma visão ecológica. Curitiba: UFPR, 2004. p.363-390.

GARRIDO, L.R.; SÔNEGO, O.R. Doenças do pessegueiro na região da serra gaúcha. Bento Gonçalves: EMBRAPA - UVA E VINHO, 2005. (Circular técnica, 61).

JENKINS, P.T.; REINGANUM, C. The occurrence of a quiescent infection of stone fruits caused by Sclerotinia fructicola (Wint.) Rehm. Australian Journal of Agricultural Research, Collingwood, v.16, p.131-140, 1965.

KISHINO, A.Y.; TSUNETA, M.; CARVALHO, S.L.C. Práticas culturais: manual agropecuário para o Paraná. Londrina-PR: Fundação Instituto Agronômico do Paraná, 1978. p.433 - 442.

MARGARIDO, S.M.F. Pêssego e nectarina: beleza e delícias no pomar. São Paulo: Ícone, 1988. 104 p.

MAY DE MIO, L.L.; GARRIDO, L.; UENO, B. Doenças de fruteiras de caroço. In: MONTEIRO, L.B.; MAY DEMIO, L.L.; SERRAT, B.M.; MOTTA, A.C.; CUQUEL, F.L. Fruteiras de caroço: uma visão ecológica. Curitiba: UFPR, 2004.p. 169 - 221.

MARTINS, M.C. Quantificação dos parâmetros monolíticos e controle químico da ferrugem do pessegueiro. 1994. 64f . Tese (Mestrado) - Escola Superior de Agricultura "Luiz de Queiroz”, Universidade de São Paulo, Piracicaba, 1994.

MARSCHNER, H. Mineral nutrition of higher plants. $2^{\text {nd }} \mathrm{ed}$. San Diego: Academic Press, 1995. 889p.

MONTEIRO, L.B.; MAY DE MIO, L.L.; MOREIRA, L.M. Monitoramento de pragas e avaliação de doenças em fruteiras de caroço. In: MONTEIRO, L.B.; MAY DEMIO, L.L.; SERRAT, B.M. MOTTA, A.C.; CUQUEL, F.L. Fruteiras de caroço: uma visão ecológica. Curitiba: UFPR, 2004. p.135-167.

NORMAS DE PRODUÇÃO INTEGRADA DE PÊSSEGO(PIP): Versão II. Pelotas: UFPel/ Ed. Pelotas: Ufpel/ Embrapa/ Ufrgs/ Urcamp, 2003. 55 p.

OGAWA, J.M.; ZEHR, E.I.; BIRD, G.W.; RITCHIE, D.F.; URIU, K.; UYEMOTO, J.K. Compendium of stone fruit diseases. St. Paul: APS Press, 1995.98 p.

PEREIRA, J.C.R.; SILVA-ACUÑA, R.; PEREIRA, A.A.; GUIMARÃES, F.B. Efeito de fontes de nitrogênio em componentes de resistência à ferrugem do cafeeiro. Revista 
Brasileira de Fitopatologia, Brasília, v.21, p.292-295, 1996.

RODRIGUES, F.A.; CARVALHO, E.M.; VALE, F.X.R. Severidade da podridão-radicular de Rhizoctonia do feijoeiro influenciado pela calagem, e pelas fontes e doses de nitrogênio. Pesquisa Agropecuária Brasileira, Brasília, v.37, p.1247-1252, 2002.

SOAVE, J.; FURLANI, P.R.; AZZINI, L.E. Relação entre o estado nutricional do arroz (Oryza sativa L.) e a suscetibilidade a Pyricularia oryzae CAV., agente causal do brusone. Summa Phytopathologica, Botucatu, v.3, p.117-123, 1977.

TANAKA, M.A.S.; FREITAS, J.G.; MEDINA, P.F.; CANTARELLA, H.; CAMARGO, C.E.O.; FERREIRAFILHO,A.W.P.; FELÍCIO, J.C. Efeito da adubação nitrogenda sobre a incidência de fungos em sementes de trigo (Triticum aestivum). Summa Phytopathologica, Botucatu, v.26, p.331-335, 2000.

TAYLOR, B.K.; VAN RAIJ, B.; The nutrition of the peach tree. IV. Storage and mobililization of nitrogen in mature trees. Australian Journal of Agricultural Research, Collingwood, v.20, p.869-881, 2003.

TUTIDA, I. Influência do nitrogênio e do potássio na intensidade de doenças fúngicas e na qualidade dos frutos em ameixeira. 2006. 71 f. Tese (Mestrado) - Universidade Federal do Paraná, Curitiba, 2006.

VAN RAIJ, B. Fertilidade do solo e adubação. São Paulo: Ed. Agronômica Ceres, 1991.p.163-176.

YAMADA, T. Resistência de plantas a pragas e doenças. Informações Agronômicas, Piracicaba, n.108, p.1-7, 2004. 